Correspondence to J.H.F.P. Department of Microbiology and Immunology, University of Maryland School of Medicine, Baltimore, Maryland 21201, USA. jpedra@som.umaryland.edu *These authors contributed equally to this work.

NEW TECHNOLOGIES: METHODS AND APPLICATIONS

\title{
Engineering of obligate intracellular bacteria: progress, challenges and paradigms
}

\author{
Erin E. McClure', Adela S. Oliva Chávez', Dana K. Shaw', Jason A. Carlyon², \\ Roman R. Ganta ${ }^{3}$, Susan M. Noh', David O. Wood ${ }^{5}$, Patrik M. Bavoil ${ }^{6}$, Kelly A. Brayton ${ }^{7}$, \\ Juan J. Martinez ${ }^{8}$, Jere W. McBride ${ }^{9}$, Raphael H. Valdivia ${ }^{10}$, Ulrike G. Munderloh ${ }^{11}$ * \\ and Joao H. F. Pedra ${ }^{*}$
}

Abstract | It is estimated that approximately one billion people are at risk of infection with obligate intracellular bacteria, but little is known about the underlying mechanisms that govern their life cycles. The difficulty in studying Chlamydia spp., Coxiella spp., Rickettsia spp., Anaplasma spp., Ehrlichia spp. and Orientia spp. is, in part, due to their genetic intractability. Recently, genetic tools have been developed; however, optimizing the genomic manipulation of obligate intracellular bacteria remains challenging. In this Review, we describe the progress in, as well as the constraints that hinder, the systematic development of a genetic toolbox for obligate intracellular bacteria. We highlight how the use of genetically manipulated pathogens has facilitated a better understanding of microbial pathogenesis and immunity, and how the engineering of obligate intracellular bacteria could enable the discovery of novel signalling circuits in host-pathogen interactions.

Bacteria have historically been divided into two distinct groups: extracellular bacteria, which exist as free-living organisms in their environmental niches, and intracellular bacteria, which infect and replicate inside host cells. Facultative intracellular bacteria, including Salmonella spp., Francisella spp., Legionella pneumophila, Listeria monocytogenes, Yersinia spp. and many others, can replicate in either niche. Conversely, obligate intracellular bacteria, including Chlamydia spp., members of the order Rickettsiales (Anaplasma spp., Ehrlichia spp., Rickettsia spp. and Orientia spp.) and Coxiella burnetii, generally require a host cell for replication (BOX 1).

Pathogenic obligate intracellular bacteria pose a substantial public health threat. In this Review, we describe, in detail, the best-studied and clinically relevant pathogens (TABLE 1), which include Coxiella spp., pathogenic Chlamydia spp. and arthropod-transmitted members of the Rickettsiales. For decades, facultative intracellular bacteria, such as Salmonella spp., Francisella spp. and L. pneumophila, have been used as models to study hostpathogen interactions ${ }^{1}$, whereas obligate intracellular bacteria have been underappreciated, despite their requirement to silently establish a replicative niche and remodel the host environment. By infecting host cells, cell biologists and immunologists can uncover novel signalling cascades that have remained uncharacterized, in part, owing to the historical focus on facultative intracellular bacteria ${ }^{2}$. Thus, obligate intracellular bacteria provide an unparalleled opportunity to discover novel principles of both pathogen and host biology.

Compared with facultative intracellular bacteria, relatively little is known about the underlying principles of bacterial physiology, host-pathogen interactions and the mechanisms that govern infection by obligate intracellular bacteria. Among the reasons for this discrepancy is the difficulty in studying bacterial signalling networks in vivo. For arthropod-transmitted members of the Rickettsiales, a lack of a basic understanding of vector physiology, immune response and microbial interactions also remains a major hurdle. More than 20 years have passed since the first attempt to transform an obligate intracellular bacterium ${ }^{3}$ (FIG. 1). The refinement of genetic manipulation methods for all obligate intracellular bacteria has proven exceedingly difficult, mostly because these microorganisms only proliferate inside of host cells. Their limited genetic toolbox often precludes the sophisticated structure-function analyses that are routinely carried out in facultative intracellular bacteria and hinders progress in public health and basic science. In this Review article, we provide an overview of the advances in, and challenges of, genetically engineering obligate intracellular bacteria. We also highlight 
Transformation

A technique that induces

bacteria to take up exogenous

DNA molecules, usually

through chemical or electrical

methods.

Polyamidoamine

dendrimers

(PAMAM dendrimers). Highly

branched polymers that can be

used to deliver small molecules

or DNA to cells. examples in which the use of genetically manipulated pathogens has improved our understanding of microbial pathogenesis and immunity. Finally, we provide an outlook on the most pressing scientific issues that could be addressed in regard to host-pathogen interactions using engineered obligate intracellular bacteria.

\section{Genetic tools: methods and limitations}

Until recently, the intracellular lifestyle of obligate intracellular bacteria has precluded the development of practical genetic tools. Prior to 2009, when an axenic medium that supports extracellular growth was developed, genetic tools for C. burnetii were limited ${ }^{4}$ (FIG. 1). Sophisticated genetic tools were rapidly developed once extracellular growth was possible, owing to the faster growth rate and ease of selecting clonal transformants ${ }^{5}$. Chlamydia spp. and members of the Rickettsiales must still be cultured and manipulated intracellularly, which poses substantial technical hurdles. In addition, obligate intracellular bacteria often grow slower than facultative intracellular bacteria. For example, Rickettsia prowazekii has a replication time of $8-12 \mathrm{~h}$, which is 2-3 times longer than L. pneumophila ${ }^{6}$. Obligate intracellular bacteria must also be purified from host cells before most transformation methods can be applied, because chemical reagents typically cannot cross both host and pathogen membranes. The purification steps are laborious, inefficient and tend to damage bacteria, which might render them non-infectious. As mutants must be selected and propagated in host cells, mutating genes that are essential for cell invasion and survival remains problematic. Last, complementation of a mutated gene with the wild-type gene under the control of its own promoter remains a major bottleneck that has only been overcome for three obligate intracellular bacteria: C. burnetii ${ }^{7}$, Chlamydia trachomatis serovar L2 (REFS 8,9) and Rickettsia parkeri ${ }^{10}$. Despite these

\footnotetext{
Author addresses

1Department of Microbiology and Immunology, University of Maryland School of Medicine, Baltimore, Maryland 21201, USA.

${ }^{2}$ Department of Microbiology and Immunology, Virginia Commonwealth University School of Medicine, Richmond, Virginia 23298, USA.

${ }^{3}$ Center of Excellence for Vector-Borne Diseases, Department of Diagnostic

Medicine/Pathobiology, College of Veterinary Medicine, Kansas State University, Manhattan, Kansas 66506, USA.

${ }^{4}$ Animal Disease Research Unit, Agricultural Research Service, U.S. Department of Agriculture and the Paul G. Allen School for Global Animal Health, Washington State University, Pullman, Washington 99164, USA.

${ }^{5}$ Department of Microbiology and Immunology, University of South Alabama College of Medicine, Mobile, Alabama 36688, USA.

${ }^{6}$ Department of Microbial Pathogenesis, University of Maryland School of Dentistry, Baltimore, Maryland 21201, USA.

'Department of Veterinary Microbiology and Pathology and the Paul G. Allen School for Global Animal Health, Washington State University, Pullman, Washington, 99164, USA.

${ }^{8}$ Vector Borne Disease Laboratories, Department of Pathobiological Sciences, Louisiana State University School of Veterinary Medicine, Baton Rouge, Louisiana 70803, USA.

${ }^{9}$ Department of Pathology, University of Texas Medical Branch, Galveston, Texas 77555, USA.

${ }^{10}$ Department for Molecular Genetics and Microbiology, Duke University, Durham,

North Carolina 27710, USA.

${ }^{11}$ Department of Entomology, University of Minnesota, St. Paul, Minnesota 55108, USA.
}

difficulties, there has been substantial progress in the development of genetic tools for obligate intracellular bacteria (BOX 2).

Transformation methods. The first step in almost all methods of genetic manipulation is transformation. Three transformation methods have been reported: chemical transformation, electroporation and polyamidoamine dendrimers (PAMAM dendrimers) mixed with a plasmid vector (FIG. 2a). Chemical transformation in a solution of calcium chloride has been used routinely to transform C. trachomatis serovar L2 since the development of a groundbreaking standard protocol in 2011 (REF. 11) (FIG. 1). Other obligate intracellular bacteria are transformed through the electroporation of cell-free bacteria ${ }^{3,5,12-15}$. Following transformation, bacteria are mixed with host cells that they must infect to survive. As chemical transformation or electroporation requires cell-free infectious bacteria, efficiency and the rate of mutant recovery would probably be improved if obligate intracellular bacteria could be manipulated within the host cell. Thus, attempts have been made to transform Chlamydia spp. and Anaplasma phagocytophilum with PAMAM dendrimers (FIC. 2a).

In proof-of-principle experiments, complexes comprising plasmid DNA and PAMAM dendrimers were added directly to infected host cell monolayers to reintroduce the cloned C. trachomatis plasmid into two plasmid-free strains, C. trachomatis serovar L2 $(25667 \mathrm{R})^{16}$ and Chlamydia pneumoniae AR-39 (REF. 17). These chlamydial transformants were stable over several passages. In addition, fluorescein isothiocyanateconjugated (FITC) dendrimers were evaluated for their ability to transform RF/6A primate endothelial cells infected with A. phagocytophilum; indeed, vacuoles that contained fluorescent $A$. phagocytophilum indicated that the dendrimers penetrated the host cell and the vacuole. In subsequent experiments, intracellular A. phagocytophilum was transformed to express GFP that was optimized for excitation by ultraviolet light (GFPuv) through dendrimers that were complexed with the transforming plasmid that integrated into the bacterial genome. However, these transformants were only retained for a few passages in medium that contained antibiotics, possibly owing to the disruption of essential genes ${ }^{18}$. Although dendrimer-enabled transformation still warrants further technical development, it could enable the introduction of markers that can be used to readily identify individual mutants in a pool, thus enabling the high-throughput screening of more recalcitrant species (for example, A. phagocytophilum). Currently, dendrimers are not routinely used to transform obligate intracellular bacteria, despite the attractive possibility to transform these bacteria inside host cells.

Shuttle vectors. Shuttle vectors are important genetic tools because they can be easily manipulated in Escherichia coli and maintained for the ectopic expression of genes under investigation in C. trachomatis, Rickettsia spp. and C. burnetii. C. trachomatis shuttle 
Allelic exchange

A common method that is used to knock in, knock out or otherwise mutagenize a DNA segment that relies on homologous recombination between the wild-type gene and an exogenous DNA construct.

Fluorescence-reported allelic exchange mutagenesis (FRAEM). A method for allelic exchange in Chlamydia trachomatis serovar L2 that can be monitored byobserving fluorescent chlamydial inclusions. vectors were derived from the endogenous $7.5 \mathrm{~kb}$ plasmid of C. trachomatis serovar LGV L2 (REF. 19). This plasmid (pL2) encodes eight genes that have diverse poorly understood roles in the infection cycle of $C$. trachomatis $^{11,20}$. A pL2 variant, pSW2, contains a deletion in the first coding sequence ${ }^{20}$. These plasmids were used to generate the shuttle vectors p2TK2-SW2 (pSW2 parent) ${ }^{21}$ and $\mathrm{pBOMB} 4$ (pL2 parent) ${ }^{22}$ by fusing them to an $E$. coli origin of replication and $\beta$-lactamase (bla) for selection (FIG. 2b). p2TK2-SW2 and pBOMB4 are used for the routine ectopic expression of proteins in C. trachomatis and have been modified to include fluorescent markers, multiple cloning sites and/or tetracyclineinducible promoters ${ }^{21-25}$. Other modified versions of the pSW2 plasmid include the vector pL2dest, which was generated to enable the direct testing of potential secreted proteins with the TEM $\beta$-lactamase reporter assay ${ }^{26}$. Until recently, it was challenging to directly manipulate the chlamydial genome through allelic exchange because the transformed bacterium retained both the targeted and wild-type gene on a stably maintained plasmid ${ }^{27}$. A technical breakthrough occurred when a suicide vector was developed. The pSU6 plasmid enabled fluorescence-reported allelic exchange mutagenesis (FRAEM) in C. trachomatis by putting pgp6, which is required for plasmid maintenance, under the control of a tetracycline-induced promoter ${ }^{27}$ (FIG. 2b). Once tetracycline is removed from the medium, $p g p 6$ is no longer expressed and the plasmid is not maintained. The pSU6 suicide vector enabled routine allelic exchange, which was previously limited to small DNA segments and a few selection markers in Chlamydia psittaci $^{28}$. The utility of the pSU6 suicide vector was illustrated by the replacement of tryptophan synthase alpha subunit $(\operatorname{trp} A)$, which is involved in tryptophan biosynthesis, and three type III secretion system (T3SS) effectors with a $g f p$ and bla cassette ${ }^{27}$. For each of the mutants, exclusively GFP-fluorescing inclusions were observed in the next passage following the removal of tetracycline, which indicates the loss of the plasmid backbone (which encodes red-fluorescent mCherry) and the integration of the allelic exchange cassette into the chlamydial genome ${ }^{27}$. As the pSU6 suicide vector was only recently developed, it has not yet been widely adopted by researchers in the Chlamydia field; however, the potential to generate targeted gene knockouts in C. trachomatis serovar L2 through allelic exchange is an exciting development ${ }^{24}$.

Shuttle vectors for Rickettsia spp. were derived from three endogenous plasmids of the spotted fever group (SFG) Rickettsia amblyommatis, namely pRAM18, pRAM23 and pRAM32, although only pRAM18 has been used extensively ${ }^{29}$. These shuttle vectors contain a rifampicin resistance cassette, a fluorescent marker,

\section{Box 1 | Lifecycle of obligate intracellular bacteria}

The life cycles of Anaplasma spp. and Ehrlichia spp. (see the figure, part a) and Chlamydia spp., (see the figure, part b) are biphasic and characterized by cyclical infectious and replicative forms. Eukaryotic host cells internalize the infectious particles (step 1), termed dense core cells (for Anaplasma spp. and Ehrlichia spp.) and elementary bodies (for Chlamydia spp.). Once inside the host cell, the elementary body differentiates into the replicative morphotype (step 2), known as the reticulate cell (for Anaplasma spp. and Ehrlichia spp.)

and reticulate body (for Chlamydia spp.).

Reticulate bodies replicate inside a vacuole (step 3), in which they eventually differentiate back to the elementary body morphotype and exit the host cell through extrusion, lysis or possibly other unknown mechanisms (step 4) ${ }^{58,69}$. The life cycles of members of the Rickettsiaceae feature cytosolic replication (see the figure, parts c,d) ${ }^{66}$. Members of the Rickettsiaceae induce their uptake by host cells (step 1) and, once internalized, they must escape the phagosome (step 2) before replication. Next, cytosolic bacteria replicate and redistribute themselves intracellularly (step 3). To complete their life cycles, Rickettsia spp. lyse the host cell (step 4) or infect neighbours through intercellular spread (step 5), whereas Orientia spp. bud from the host cell

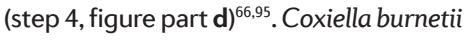
(see the figure, part e) is phagocytosed by the host (step 1). The phagosome matures into the Coxiella-containing vacuole (step 2), in which the bacteria differentiate into the large cell variant and replicate (step 3). Last, the bacteria differentiate back into the infectious small cell variant and lyse the cell (step 4) $)^{96,97}$. a Anaplasma spp. and Ehrlichia spp.
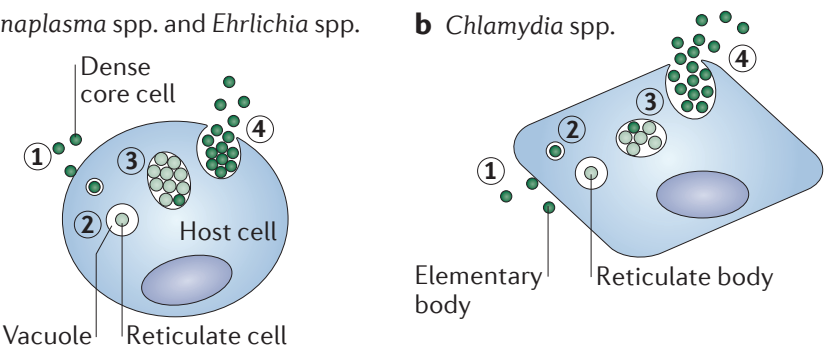

c Rickettsia spp.

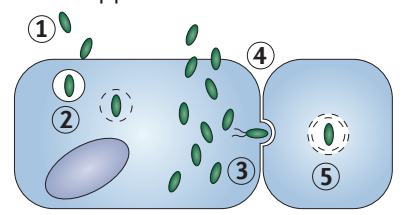

d Orientia spp.

e Coxiella burnetii

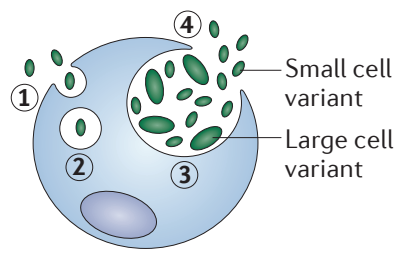

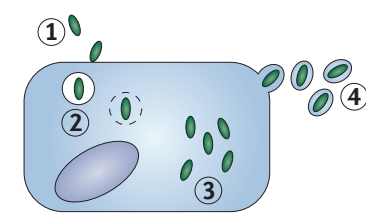


Table 1 | Disease pathogenesis of selected obligate intracellular bacteria

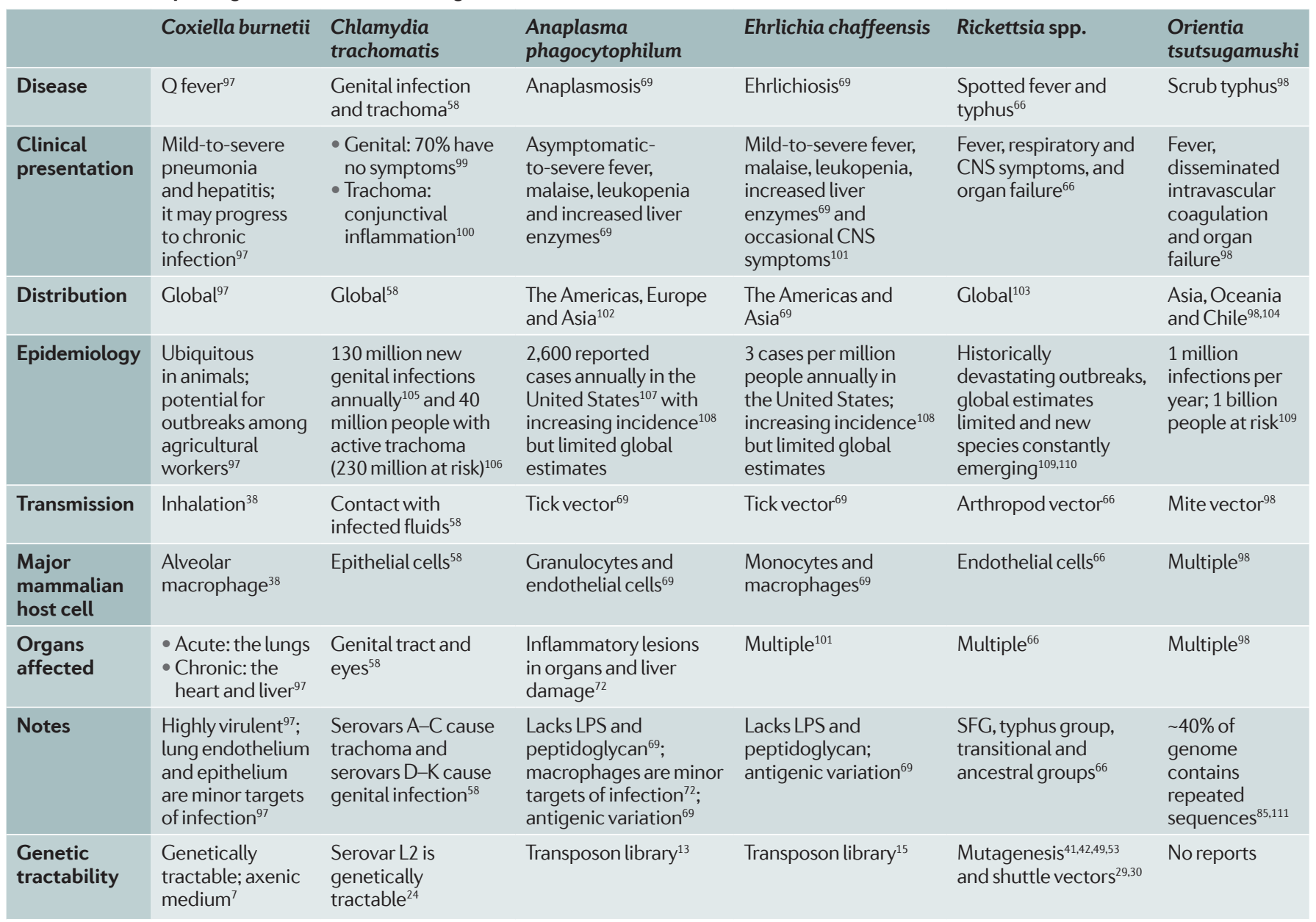

CNS, central nervous system; LPS, lipopolysaccharide; SFG, spotted fever group.

Dot/Icm type IV secretion system

A set of bacterial proteins that inject effector molecules into the eukaryotic host cytosol to remodel the intracellular niche. portions of standard $E$. coli vectors that confer antibiotic resistance and regions of the endogenous R. amblyommatis plasmids that contain $d n a A$ and par $A^{29}$, which are required for the replication and maintenance of DNA (FIG. 2b). The pRAM18dRGA and pRAM32dRGA plasmids were transformed into the SFG Rickettsia conorii, R. parkeri, Rickettsia montanensis, Rickettsia monacensis and $R$. amblyommatis, the typhus group $R$. prowazekii ${ }^{30}$ and Rickettsia typhi ${ }^{31}$, and the ancestral group Rickettsia bellii. With the exception of $R$. amblyommatis, all of the bacteria acquired a fluorescent signal, which indicated successful transformation and expression from the shuttle vector ${ }^{29}$. Failure to establish pRAM18dRGA in the parent $R$. amblyommatis confirms that plasmids that share the same partitioning system are incompatible in rickettsiae ${ }^{32}$.

A multiple cloning site was inserted into pRAM18dRGA, which facilitated the ligation of a gene that encodes the red fluorescent protein mCherry under the control of an Anaplasma marginale promoter (yielding pRAM18dRGA[AmTrCh]). Both R. montanensis and $R$. conorii were transformed with this shuttle vector and acquired red fluorescence, which demonstrates the functionality of this construct ${ }^{29,33}$. Interestingly,
R. conorii transformed with pRAM18dRGA[AmTrCh] was maintained in cell culture and within an experimentally infected animal without the administration of antibiotics ${ }^{34}$, which indicates that the plasmids were stable for the duration of these experiments in the absence of antibiotic selection. Furthermore, rick $A$ from $R$. monacensis was overexpressed in $R$. bellii from the multiple cloning site of the shuttle vector, which resulted in significant changes in adhesion to cells and intracellular motility $^{35}$. Finally, pRAM18dRGA[Sca4] complemented surface cell antigen 4 (sca4) in an R. parkeri mutant ${ }^{10}$.

Shuttle vectors for C. burnetii were created following the establishment of the axenic medium. Two reporter plasmids that enabled for $\beta$-lactamase-gene fusions, pCBTEM $^{36}$ and pJB-CAT-BlaM ${ }^{37}$, were independently generated. C. burnetii uses the Dot/lcm type IV secretion system to remodel its intracellular niche (Supplementary information S1 (figure)), and detailed knowledge of substrates was gained through TEM $\beta$-lactamase reporter assays ${ }^{38}$. The pJB-CAT plasmid backbone was used to generate other shuttle vectors that enabled the ectopic expression of tagged proteins in C. burnetii and complementation of mutated genes ${ }^{7}$. Although the copy numbers of shuttle vectors are difficult to control 


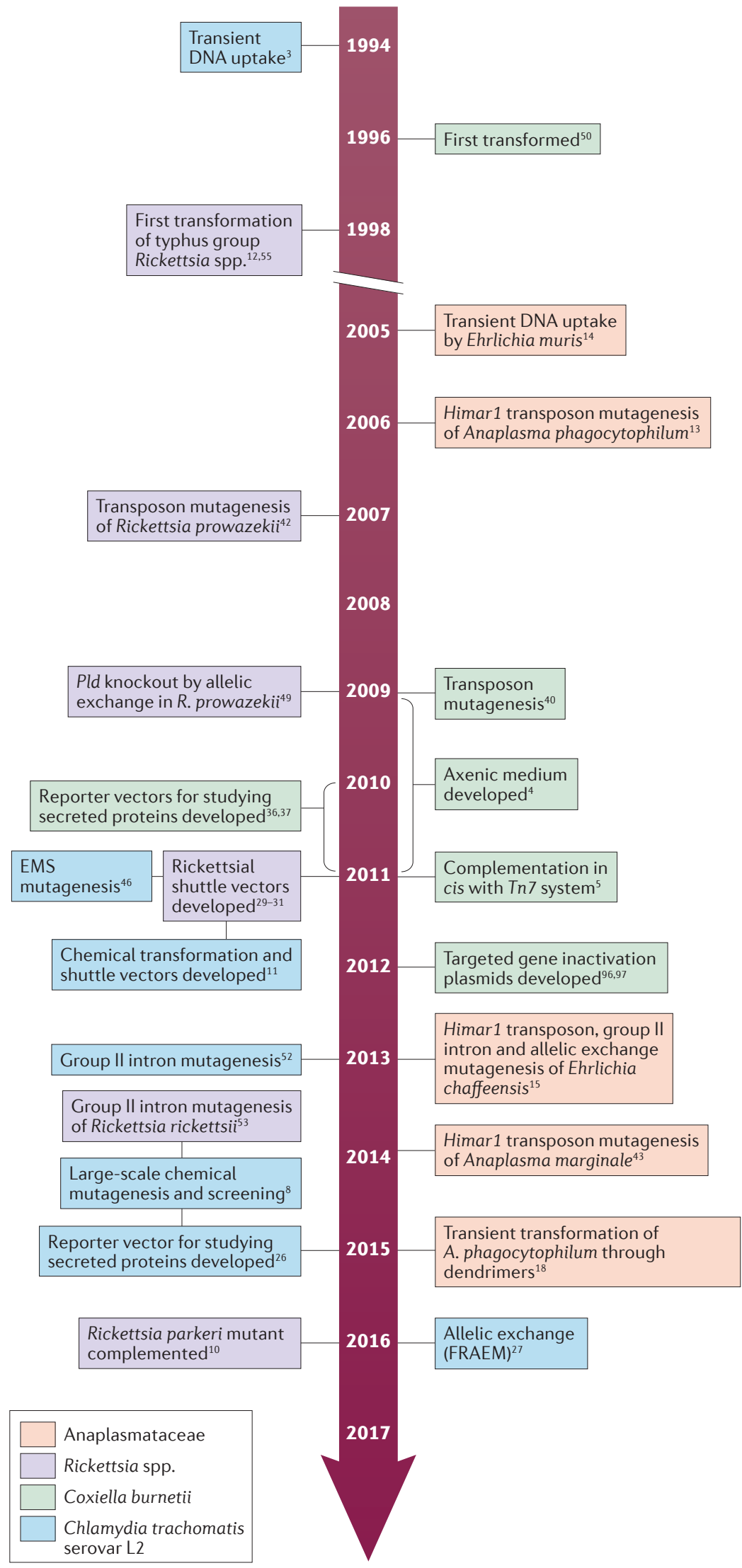

Figure 1 | Advances in the genetic manipulation of obligate intracellular bacteria. EMS, ethyl methanesulfonate; FRAEM, fluorescence-reported allelic exchange mutagenesis; Pld, phospholipase D. and may cause polar effects, they provide a convenient method to examine gene function directly in obligate intracellular bacteria.

Mutagenesis. Random mutagenesis methods include transposon insertion and chemical mutagenesis (FIG. 2C). Himar1 is a mariner transposon that is derived from a transposable element isolated from the stable fly Haematobia irritans ${ }^{39}$ that has been successfully used to mutagenize various organisms, including several obligate intracellular bacteria, such as C. burneti ${ }^{40}$, Rickettsia spp. ${ }^{41,42}$, Anaplasma spp. ${ }^{13,43}$ and Ehrlichia chaffeensis ${ }^{15}$. The Himar 1 transposase system encodes the transposase and the transposon on one or two suicide plasmids that are electroporated into host cell-free bacteria ${ }^{13,42,44}$. The Himar 1 transposase randomly integrates the transposon, which contains an antibiotic resistance cassette and fluorescent markers flanked by repeats, into AT dinucleotide sites in the bacterial genome through a cut-and-paste mechanism ${ }^{13}$. Genetic markers in the transposon facilitate selection, visual detection and the identification of insertion loci in the bacterial genome. Transposase systems have also been used to complement C. burnetii mutations in $\mathrm{cis}^{7}$.

Chemical mutagenesis provides an alternative to the Himar 1 transposon system, but without selection or detection markers. To date, only Chlamydia abortus (formerly C. psittaci var. ovis) and C. trachomatis have been successfully mutagenized using DNA-alkylating agents to generate libraries ${ }^{8,45-47}$. Chemical mutagenesis does not require bacterial transformation, and obligate intracellular bacteria do not need to be purified from the host cell before application of the mutagen. Another advantage of chemical mutagenesis is that hypomorphic mutations, which decrease gene function instead of completely inactivating it, can be generated, enabling the characterization of genes that are involved in essential functions $s^{25}$. Linking mutations to observed phenotypes can be laborious because it is technically demanding to identify, select and recover mutants in the absence of molecular tags ${ }^{24}$. Nonetheless, as Chlamydia spp. are naturally competent, carrying out linkage analysis can be carried out using lateral gene transfer ${ }^{24,25}$. Co-infection with a rifampicin-resistant mutant and a spectinomycinresistant wild-type strain produces recombinant mutants that are resistant to both antibiotics. The recombinant clones are then genotyped and assessed for phenotype $\mathrm{e}^{48}$. Repeated rounds of co-infection, phenotypic screening and genotyping are carried out until a single mutation can be linked to a phenotype. Other strategies involve using temperature-sensitive mutants to carry out genetic mapping, which does not require the generation of antibiotic-resistant strains of C. trachomatis ${ }^{47}$. Libraries of mutant $C$. trachomatis strains obtained through ethyl methanesulfonate mutagenesis (EMS mutagenesis) have been screened using various approaches, including temperature, forward genetics and reverse genetics ${ }^{46-48}$. Although linking phenotypes to mutations is tedious, chemical mutagenesis has proven to be an important technique for elucidating the function of genes in C. trachomatis serovar L2. For example, EMS mutagenesis has 
Mariner transposon

An abundant class II transposable element first discovered in Drosophila spp. that integrates into a wide range of genomes.

Ethyl methanesulfonate mutagenesis

(EMS mutagenesis). A technique in which a DNA-alkylating agent (EMS) is applied to a population of cells to create a library of strains that contain random mutations.

Mobile group II introns Mobile bacterial ribozymes that self-splice, reverse transcribe the spliced RNA into DNA, and then integrate the DNA into the bacterial chromosome. led to the characterization of chlamydial metabolic features and the identification of an effector that is responsible for mediating the assembly of filamentous actin (F-actin) around the inclusion ${ }^{8}$.

Targeted mutagenesis includes allelic exchange and mobile group II introns. Allelic exchange is routinely used to generate mutants in facultative intracellular bacteria and extracellular bacteria; only recently has this technique been successfully applied to obligate intracellular bacteria (FIG. 3a). Early attempts to mutagenize Chlamydia spp. resulted in allelic exchange of the ribosomal RNA (rRNA) operon with a synthetic 16S rRNA gene that contained point mutations, which rendered the mutant bacterium resistant to spectinomycin and kasugamycin ${ }^{28}$. This technique has only been successful in C. psittaci and has been limited to replacing small gene segments ${ }^{28}$. The recent development of the pSU6 suicide vector facilitated allelic exchange in C. trachomatis serovar L2 (REF. 27) (FIG. 2b).

Allelic exchange has also been carried out in $R$. prowazekii to inactivate the phospholipase $\mathrm{D}$ gene ( $p l d$ ), which is a putative virulence factor ${ }^{49}$. The pld ORF and surrounding bases were cloned into the pBluescript $\mathrm{SKII}^{+}$plasmid and an internal segment was replaced with a rifampicin resistance cassette ${ }^{49}$. Transformation was achieved using a linear DNA fragment that was amplified from the construct, as it was less likely to result in the insertion of the entire plasmid into the rickettsial genome ${ }^{49}$. The resulting $\Delta$ pld R. prowazekii mutant strain was used to determine whether the disruption of pld leads to attenuated virulence in $R$. prowazekii ${ }^{49}$. In E. chaffeensis, two types of recombinant linear DNA segment that targeted a hypothetical protein were generated. One segment was prepared to introduce an antibiotic resistance cassette to disrupt the gene, which resulted in an increase in the size of the bacterial genome. To avoid polar effects, a second recombinant segment was created to remove a segment of the disrupted gene, such that the resulting construct was approximately the size of the wild-type gene. Mutants were detected independently of the recombination methods, but they persisted for only a few days in culture ${ }^{15}$. Last, allelic exchange was used to transform C. burnetii to ampicillin resistance $e^{50}$, but this technique is not widely used in the Coxiella field, perhaps, in part, owing to the success of other genetic tools.

Mobile group II introns facilitate the selective integration of DNA that is reverse-transcribed from RNA into specific sites in the bacterial genome $e^{51}$. The selectivity of insertion is conferred through the sequence of the intron RNA, and commercial systems, such as TargeTron, have been successfully used to genetically modify $E$. chaffeensis, C. trachomatis serovar L2 and Rickettsia spp. through insertional mutagenesis (FIG. 3a). In E. chaffeensis, TargeTron constructs were assembled for six different genes, including those that encode outer membrane proteins ${ }^{15}$. Unfortunately, the resulting mutants were only detectable for less than a week and stable mutant lines could not be selected. By contrast, TargeTron constructs were used to mutate the C. trachomatis serovar L2 effector inclusion membrane protein $\mathrm{A}(\text { inc } A)^{52}$, which

\footnotetext{
Box 2 | Genetic tools for obligate intracellular bacteria

Although all obligate intracellular bacteria adopt intracellular lifestyles, they are not equally amenable to genetic manipulation. Coxiella burnetii is technically no longer an obligate intracellular bacterium owing to the development of an axenic medium that enabled its host-free cultivation in the laboratory ${ }^{5}$. Most genetic tools have been developed for Chlamydia trachomatis serovar L2, which is now considered to be genetically tractable ${ }^{24}$. However, genome manipulation of clinically relevant $C$. trachomatis serovars and other Chlamydia spp. remains challenging. In the order Rickettsiales, Rickettsia spp. (spotted fever group (SFG) and typhus group) have the most sophisticated genetic tools available, and a recent article was the first to report genetic complementation $^{10}$. Fewer genetic tools have been developed for members of the Anaplasmataceae family (Anaplasma spp. and Ehrlichia spp.), and no successful genetic manipulation has been reported for Orientia tsutsugamushi (in the family Rickettsiaceae). The differential ability to genetically manipulate obligate intracellular bacteria may be due, in part, to the biological differences between species (for example, the ability to form plaques or the presence of endogenous plasmids). A lack of adoption of some genetic tools may be caused by the small size of the scientific community that researches obligate intracellular bacteria, which limits the number of groups that contribute to the development and refinement of genomic techniques. In the figure, one tick indicates successful application of a genetic tool (columns) for each major species (rows). Two ticks indicate that the technique has been published by at least two independent research groups.
}

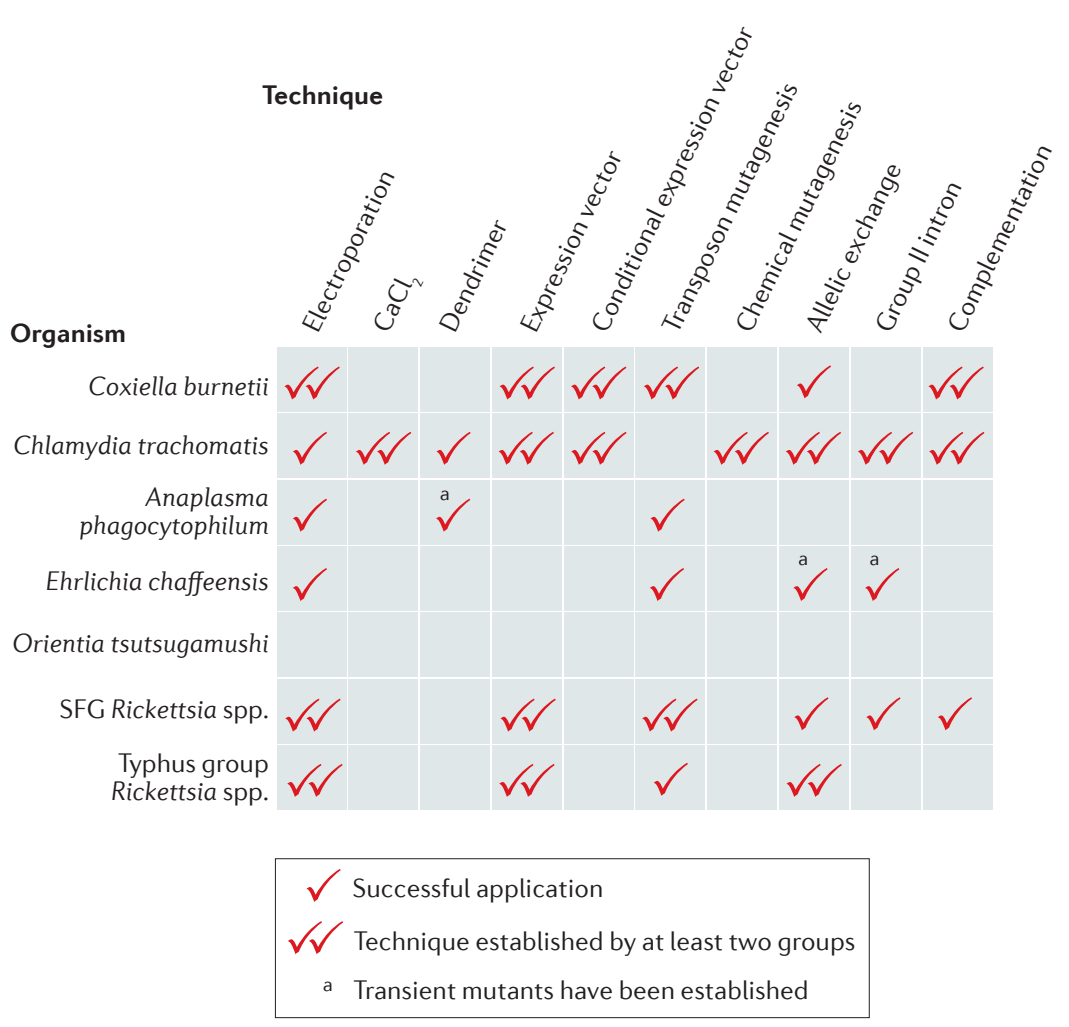




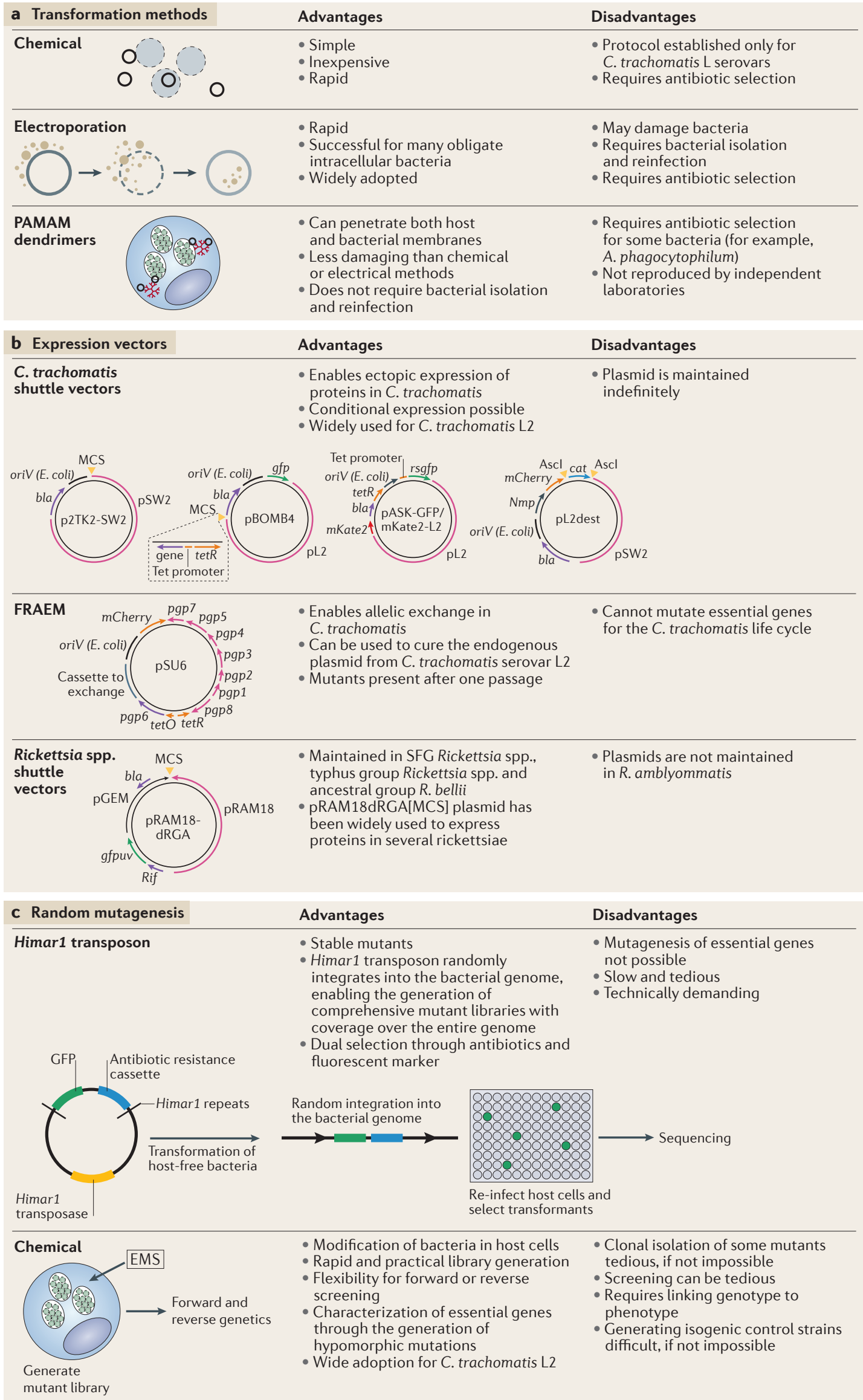


is thought to promote the fusion of inclusions inside cells that are infected with C. trachomatis ${ }^{52}$. Recently, Chlamydia promoter of cell survival (CpoS) was disrupted by TargeTron, confirming the phenotype of a chemically mutagenized strain ${ }^{9}$. In another example, a premature stop codon and a rifampicin resistance cassette were introduced to inactivate outer membrane protein $\mathrm{A}(o m p A)$ in virulent Rickettsia rickettsii str. Sheila Smith ${ }^{53}$. There are only a few cases of the successful application of TargeTron constructs to generate stable targeted mutants of obligate intracellular bacteria. This could be attributed to the proprietary TargeTron technology or the availability of other tools (for example, allelic exchange).

Recovery and selection. Three methods are currently used to distinguish mutants from the wild-type bacteria: antibiotic selection, fluorescence-activated cell sorting (FACS) and laser microdissection (FIG. 3b). Antibiotic selection is a standard technique that enables the survival of only those bacteria that acquired a resistance cassette that encodes factors that promote survival when exposed to xenobiotics. Only clinically irrelevant antibiotics are permitted for use by regulatory agencies, owing to the potential for the escape of resistant strains into the environment. These regulations further limit the small range of antibiotics that are effective against obligate intracellular bacteria. Moreover, different species, strains, biovars and serovars may be differentially susceptible to antibiotics ${ }^{24}$, and resistance can develop after continued exposure in cell culture. Cells infected with labelled C. trachomatis and Rickettsia spp. that express a fluorescent protein have been sorted by FACS ${ }^{33,54-56}$. Similarly, single cells that are infected with labelled C. trachomatis have been successfully isolated

\section{- Figure 2 |Transformation methods, expression vectors and random mutagenesis.}

a |Chemical transformation with calcium chloride is used to transform Chlamydia trachomatis serovar L2. Electroporation is primarily used to transform members of the order Rickettsiales ${ }^{13,15,33}$. Plasmids complexed with polyamidoamine (PAMAM) dendrimers provide an alternative method to transform obligate intracellular bacteria. b|Shuttle vectors have been developed for C. trachomatis and Rickettsia spp. In both cases, portions of endogenous plasmids (pSW2 and pL2 for C. trachomatis, and pRAM18 for Rickettsia spp.) are fused to Escherichia coli plasmid backbones to enable replication. The shuttle vectors include E. coli origins of replication (oriV), fluorescent markers (GFP and GFP optimized for excitation by ultraviolet light (GFPuv) or red fluorescent protein (mCherry)), antibiotic selection genes ( $\beta$-lactamase (bla), chloramphenicol (cat), spectinomycin (aadA) and rifampicin resistance (rif)) and multiple cloning sites (MCS) ${ }^{21,22,29}$. Two chlamydial shuttle vectors, pBOMB4 and pASK-GFP/mKate2-L2, can be modified to include a tetracycline-inducible promoter for conditional gene expression $^{22,23}$. The shuttle vector $\mathrm{pL2dest} \mathrm{enables} \mathrm{the} \mathrm{expression} \mathrm{of} \mathrm{proteins} \mathrm{that} \mathrm{are}$ fused with a $\beta$-lactamase reporter to study protein secretion ${ }^{26}$. The fluorescencereported allelic exchange mutagenesis (FRAEM) vector pSU6 enables allelic exchange in C. trachomatis by behaving as a suicide vector in the absence of tetracycline ${ }^{27}$. c| The Himar1 transposase randomly inserts the transposon into the bacterial genome. Successful transformants are selected with antibiotics and the expression of a fluorescent protein ${ }^{13}$. The mutants are selected and sequenced to determine insertion sites. In chemical mutagenesis, mutations are caused by DNA-alkylating agents (for example, ethyl methanesulfonate (EMS)). Mutants are selected, pooled and subjected to forward or reverse genetics screens ${ }^{8}$. A. phagocytophilum, Anaplasma phagocytophilum; R. amblyommatis, Rickettsia amblyommatis; R. bellii, Rickettsia bellii; SFG, spotted fever group; tet $O$, tetracycline operator; tet $R$, tetracycline repressor. through laser microdissection ${ }^{57}$. However, the technical demands and cost of specialized equipment, coupled with biosafety limitations (for example, maintenance of cell sorters in biosafety level 3 laboratories), prevent these techniques from being widely used. Although the selection of transformants can be tedious and potentially expensive, a major hurdle to overcome is the isolation of clonal mutants. Micromanipulation of cells that are infected with C. burnetii has been used to extract single Coxiella-containing vacuoles, which can then infect sterile host cells or be cultivated in axenic medium ${ }^{5,40}$. As there are no axenic media available for most obligate intracellular bacteria, simple clone picking and expansion are not possible. Some strains of Chlamydia spp. and Rickettsia spp. can be plaque purified to obtain clonal transformants, but not all obligate intracellular bacteria form visible plaques. Therefore, this method is not widely applicable ${ }^{5}$. Alternatively, clonal transformants can be recovered through time-consuming limiting dilution $^{5}$ (FIG. 3b).

\section{New functional insights}

Chlamydia trachomatis effectors. Chlamydia spp. reside in a membrane-bound inclusion that becomes remodelled by integral membrane proteins (Supplementary information S1 (figure)). These inclusion proteins (Incs) are secreted by the T3SS and regulate membrane trafficking, cell signalling and cytoskeletal rearrangements to promote the formation and maintenance of inclusions $^{58}$. C. trachomatis has more than 50 putative Inc proteins, some of which are expressed at unique stages of the developmental cycle ${ }^{59}$.

Despite the importance of Incs for infection with Chlamydia spp., detailed knowledge at the mechanistic level was limited until recently, owing to the genetic intractability of these microorganisms. Recently, a mutant library of approximately 1,000 EMS-mutagenized isolates of C. trachomatis was generated $^{8}$. A microscopy-based forward-genetics screen was used to identify two mutants that had abnormal F-actin assembly, as deficits in this function were likely due to mutations in proteins that control inclusion maturation. The sequencing of one mutant (strain M407) and genetic linkage analysis led to the identification of a nonsense mutation in CTL0184 (C307T/Q103*). The assembly of F-actin was restored in strain M407 complemented with the p2TK2-SW2 shuttle vector that encoded wild-type CTL0184 under the control of its endogenous promoter.

CTL0184 was subsequently investigated and renamed inclusion membrane protein for actin assembly (InaC; FIG. 4a). Co-immunoprecipitation assays identified two classes of host molecule, 14-3-3 scaffolding proteins and ADP-ribosylation factors (ADPribosylation factor 1 (ARF1), ARF4 and ARF5), as InaC interacting partners. InaC and F-actin assembly were shown to be required for the redistribution of the Golgi body, which is thought to be a source of lipids for inclusion remodelling ${ }^{58}$. ARFs are Golgi-associated GTPases that regulate membrane trafficking ${ }^{60}$. However, the InaC-mediated enlistment of ARFs was not important 
for lipid acquisition in the cell culture system ${ }^{8}$, and the mechanism by which InaC regulates the assembly of F-actin and Golgi distribution together with ARFs and 14-3-3 proteins remains to be identified. Other intracellular pathogens, such as L. pneumophila and typhus group Rickettsia spp., recruit host ARFs through their RalF effector proteins ${ }^{61-63}$. L. pneumophila uses ARF1 to control the delivery of endoplasmic reticulum

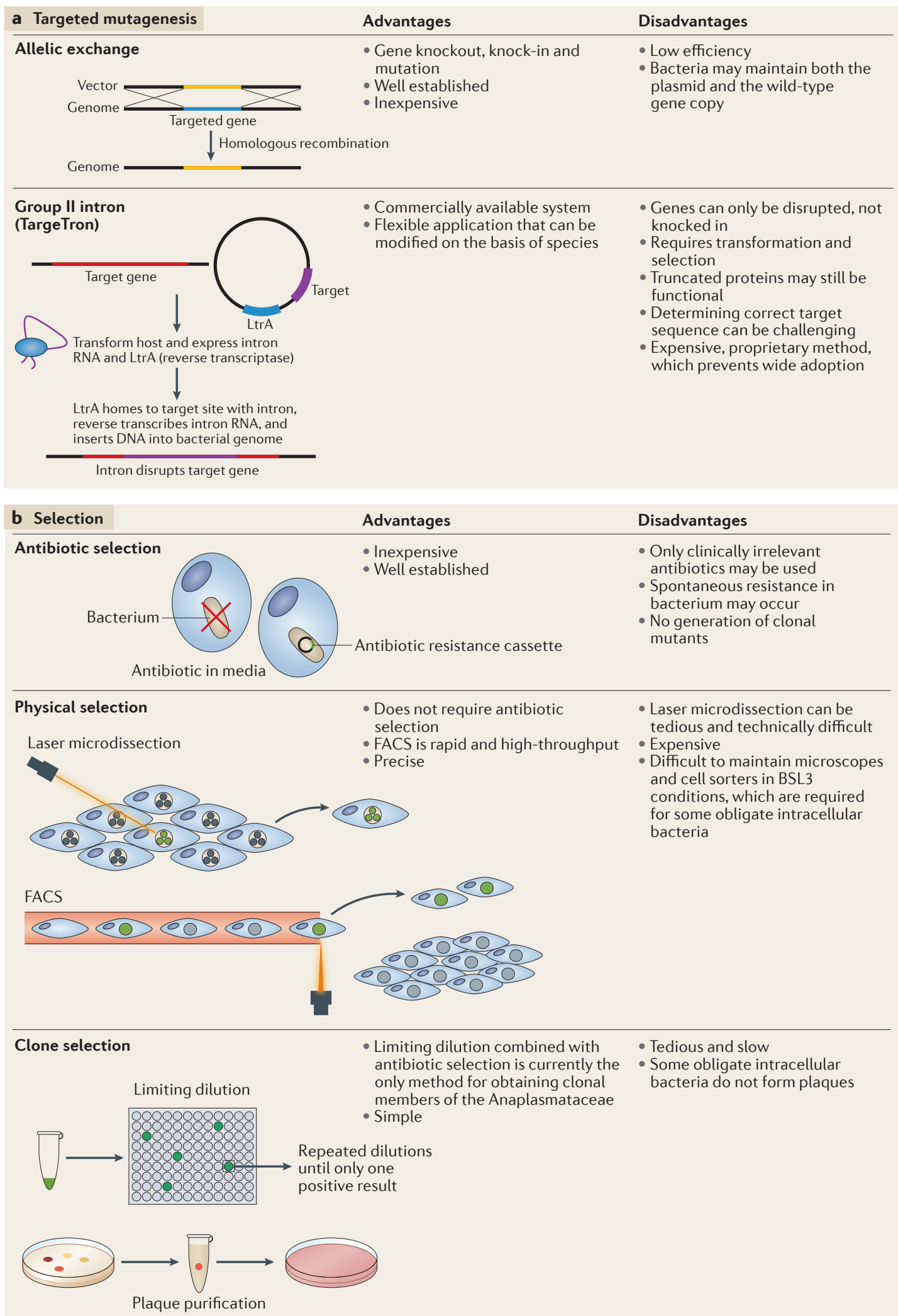


Necrosis

A type of inflammatory cell death that occurs

spontaneously after damage to a cell.

\section{Apoptosis}

A mode of non-inflammatory programmed cell death

Stimulator of interferon genes

(STING). An endoplasmic reticulum-associated cytosolic intracellular pattern recognition molecule that senses cyclic dinucleotides and induces the production of type I interferons

Vinculin

A mammalian cytoskeletal protein that anchors the cell membrane to the actin cytoskeleton.

Granulocytic anaplasmosis A mild-to-severe tick-borne infectious disease caused by Anaplasma phagocytophilum, which infects neutrophils and myeloid cells, that is characterized by fever, thrombocytopenia, leukopenia and liver damage.
(ER)-derived vesicles to the maturing Legionellacontaining vacuole ${ }^{63}$. Interestingly, the typhus group Rickettsia species $R$. prowazekii ${ }^{61}$ and $R$. typhi hijack ARFs to manipulate the rearrangement of the actin cytoskeleton at the plasma membrane, which has been shown to lead to pathogen uptake in R. typhi ${ }^{62}$. Thus, it is likely that C. trachomatis, similarly to other intracellular pathogens, uses ARFs to manipulate host vesicle trafficking and actin cytoskeleton dynamics during the establishment of the inclusion.

The Inc protein CTL0481, which was renamed Chlamydia promoter of survival (CpoS), was also recently identified in a forward genetic screen for C. trachomatis mutants that enhanced the death of infected cells ${ }^{9}$. Loss-of-function mutations in $c p o S$ led tothe induction of necrosis and apoptosis midway through the infection cycle, which correlated with an exaggerated type I interferon (IFN) response. In agreement with this, complementation of the chemically mutagenized strain with a p2TK2-SW2-derived plasmid that encodes cpos did not elicit host cell death. Furthermore, inactivation of $c p o S$ using a TargeTron construct in a wild-type strain of $C$. trachomatis serovar L2 induced cell death. Unexpectedly, the release of type I IFNs was uncoupled from cell death, although the cyclic dinucleotide sensor stimulator of interferon genes (STING) was required. The interacting partners of CpoS included multiple RAB proteins that control ER-to-Golgi protein trafficking, which suggests that it might modulate membrane trafficking at the inclusion? Last, as STING and calcium flux are suggested to be linked ${ }^{64,65}$, and the depletion of ER calcium stores partially rescued the cell death phenotype of CpoS-deficient mutants, CpoS-mediated STING and cell death inhibition may be attributed to calcium homeostasis ${ }^{9}$.

\section{Rickettsial dissemination, $A$. phagocytophilum moon- lighting and $\mathrm{E}$. chaffeensis growth. SFG rickettsiae are cytosolic obligate intracellular bacteria that cause vascular and endothelial tissue damage ${ }^{66}$ (TABLE 1; Supplementary information S1 (figure)). They dissem- inate intracellularly in the nutrient-rich host cytosol and move from cell to cell without entering the extra- cellular environment ${ }^{10}$. Largely on the basis of electron microscopy and studies in L. monocytogenes, it was assumed that SFG rickettsiae co-opted host actin with a single bacterial effector to propel themselves through}

\footnotetext{
Figure $3 \mid$ Targeted mutagenesis and selection. a |Targeted mutagenesis enables the alteration of specific bacterial genes. Allelic exchange can be used to introduce point mutations, and to insert and delete specific genes. Group II intron technology (TargeTron) enables introns to be specifically inserted into bacterial genes to disrupt gene function through LtrA, a multifunctional protein derived from Lactococcus lactis, which reverse transcribes and splices the intron and cleaves the recipient DNA for intron insertion ${ }^{51}$. TargeTrons have been successfully applied to generate transient mutants of Ehrlichia chaffeensis ${ }^{15}$ and stable mutants of Chlamydia trachomatis and Rickettsia rickettsii ${ }^{52,53}$. b | Two methods are currently used to distinguish mutants from wild-type bacteria: antibiotic selection and physical selection. Mutants can be physically separated from wild-type bacteria by fluorescence-activated cell sorting (FACS) and laser microdissection. One final step is obtaining clonal mutants, which can be isolated by limiting dilution, or, in some cases, plaque purification ${ }^{5}$. BSL3, biological safety level 3.
}

plasma membranes into a recipient cell, in which they quickly escaped into the cytoplasm ${ }^{10}$. However, $R$. parkeri mutants that are defective in actin-based motility revealed that $R$. parkeri uses two different actin-polymerizing proteins, RickA and Sca2, which are not used by other motile intracellular bacteria ${ }^{41}$. This, together with basic phenotypic differences with the L. monocytogenes model (such as a shorter time in protrusions, a lack of actin tails and a quicker transition from protrusion to vesicle), suggests an alternative mechanism for intercellular spread ${ }^{10}$.

A Himar1 mutagenesis library of $R$. parkeri was screened for mutants that formed small plaques, as these were likely to be deficient in some aspect of intercellular spread ${ }^{10,41}$ (FIG. 4b). One mutant had a transposon insertion in sca4, which led to the production of a truncated Sca4 protein ${ }^{10}$. Sca4 was predicted to be secreted ${ }^{67}$ and shown to bind to vinculin ${ }^{68}$, but its role in infection remained unclear. A series of traction force microscopy experiments showed that Sca4 decreased intercellular tension to enable $R$. parkeri to spread into the donor cell independently of actin-based motility ${ }^{10}$ (FIG. 4b). Importantly, normal intercellular spread of sca4::Himar1 was restored after complementation with the pRAM18dRGA[Sca4] expression vector ${ }^{10}$.

A. phagocytophilum is a tick-borne pathogen that causes granulocytic anaplasmosis in humans (HGA) and other mammals ${ }^{69}$ (TABLE 1; Supplementary information S1 (figure)). Himar1 transposon mutagenesis enabled the generation of a mutant library of A. phagocytophilum to study disease pathogenesis ${ }^{13}$. Library screening led to the identification of an A. phagocytophilum mutant that contains a single transposon insertion in the dihydrolipoamide dehydrogenase 1 (lpda1) gene $\mathrm{e}^{70}$ (FIC. 4C), which encodes a metabolic enzyme that has been implicated to have alternate functions in virulence ${ }^{71}$. In a murine model of infection, the $A$. phagocytophilum mutant $l p d a 1::$ Himar 1 induced several clinical abnormalities, including more pronounced peripheral blood neutropenia and higher erythrocyte count ${ }^{70}$. Increased levels of IFN $\gamma$, splenomegaly and splenic extramedullary haematopoiesis were observed in mice that were infected with the lpda1::Himar1 mutant strain but not with the wild-type bacterium. Transient infection of macrophages by the Ipda1::Himar1 A. phagocytophilum strain correlated with enhanced nuclear factor- $\kappa \mathrm{B}(\mathrm{NF}-\kappa \mathrm{B})$ activity, which led to an increase in the production of reactive oxygen species (ROS) and the release of pro-inflammatory cytokines ${ }^{70}$. Thus, the normal expression of LpdA1 in wild-type bacteria may prevent enhanced immune activation in macrophages, which are increasingly being recognized as the immune cells that are responsible for the cytokine storm that is characteristic of A. phagocytophilum infection ${ }^{72}$. These findings suggest that protein moonlighting, a phenomenon whereby a protein carries out more than one function ${ }^{71}$, may be used as a strategy of immune evasion by A. phagocytophilum.

Transposon-based mutagenesis of E. chaffeensis, an emerging tick-borne pathogen, has become valuable for vaccine development (TABLE 1; Supplementary information S1 (figure)). This approach was used to create 
a Chlamydia trachomatis: InaC

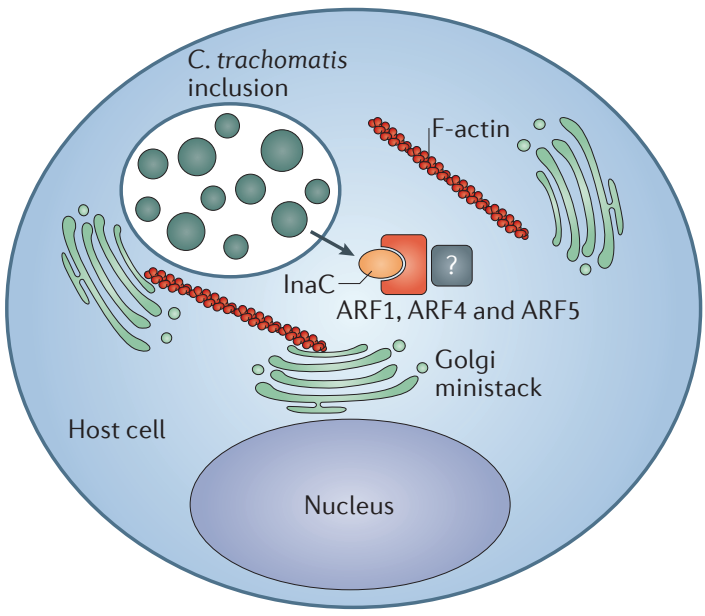

b Rickettsia parkeri: Sca4

Defective intercellular spread

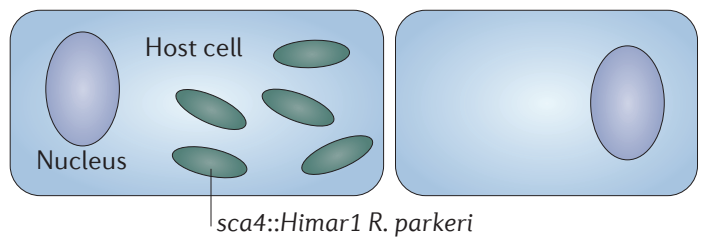

Restored intercellular spread

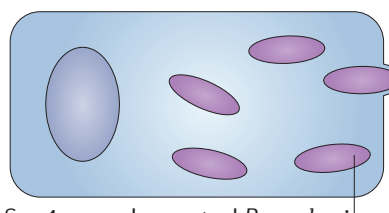

Sca4-complemented R. parkeri

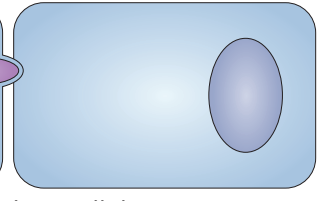

Intercellular tension reduced by Sca4 c Anaplasma phagocytophilum: LPDA1
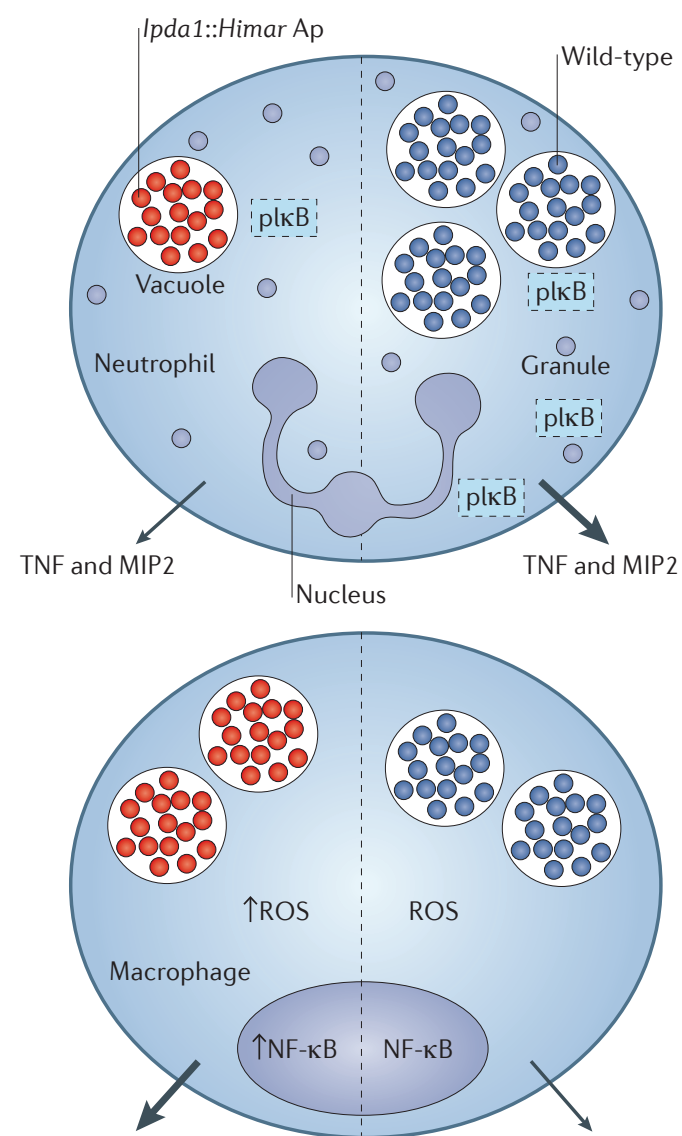

TNF and IL-12p40

TNF and IL-12p40

Figure 4 | Discoveries in microbial pathogenesis facilitated by genetic tools. a | Chemical mutagenesis of Chlamydia trachomatis led to the identification of inclusion membrane protein for actin assembly (InaC), which recruits filamentous actin (F-actin) and induces Golgi redistribution around the inclusion in manner that is dependent on ADP ribosylation factor 1 (ARF1), ARF4 and ARF5 (REF. 8). b| Rickettsia parkeri disseminates intercellularly through a mechanism that is not used by facultative intracellular bacteria. Screening of a Himar1 transposon mutagenesis library of R. parkeri identified a mutant deficient in intercellular spread named surface cell antigen 4 (sca4)::Himar1 (REF. 10). The sca4::Himar1 mutant was complemented by pRAM18dRGA[Sca4], which restored intercellular spread. Sca4 was found to inhibit vinculin binding toa-catenin (not shown), thereby reducing intercellular force transduction and enabling the intercellular spread of R. parkeri.c| Himar1 transposon mutagenesis was used to generate an Anaplasma phagocytophilum mutant library. One mutant exhibited a single transposon insertion in the dihydrolipoamide dehydrogenase 1 (lpda1) gene. Compared with wild-type infection, the lpda1::Himar1 A. phagocytophilum mutant infected neutrophils less well, and activated nuclear

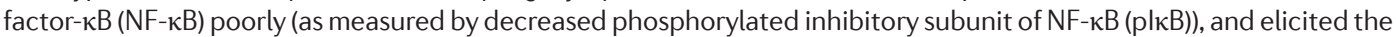
production of less tumour necrosis factor (TNF) and macrophage inflammatory protein 2 (MIP2; also known as CXCL2) in neutrophils ${ }^{70}$. Transient infection of macrophages by the lpda1::Himar1 A. phagocytophilum strain correlated with enhanced nuclear NF-kB activity, which led to the increased production of reactive oxygen species (ROS) and the release of pro-inflammatory cytokines ${ }^{70}$. Mice that were infected with the lpda1::Himar1 mutant or wild-type A. phagocytophilum $\mathrm{HZ}$ exhibited differences in immunopathology and cell-specific outcomes, which suggests that LPDA1 may have a role in inhibiting excessive host immune activation ${ }^{70}$. Arrow size correlates with relative quantity of cytokines secreted. IL-12p40, interleukin-12 subunit $\mathrm{p} 40$.

mutations in four genes that are predicted to encode membrane proteins (Ech_0230,Ech_0379,Ech_0601 and Ech_0660 $)^{15}$. With the exception of Ech_0601, all mutations caused attenuation of the growth of the pathogen in both the reservoir (deer) and incidental (dog) hosts. Conversely, no effect on bacterial growth was observed in the tick vector Amblyomma americanum ${ }^{73}$.
Two clonally-purified attenuated mutants that had insertions in the Ech_0379 and Ech_0660 genes provided protection against wild-type infection in both deer and $\operatorname{dogs} \mathrm{s}^{74}$. Thus, refining genetic tools for practical application in obligate intracellular bacteria could accelerate vaccine development and protect vulnerable populations. 
Correcting virulence factor paradigms. Classified as a select agent, $R$. prowazekii is considered a potential bioweapon and no vaccine against $R$. prowazekii exists in the United States; hence, research into the virulence of $R$. prowazekii that leads to an effective vaccine is vital to protect public health ${ }^{49}$. Comparative genomics approaches that examined the differences between virulent and avirulent strains of $R$. prowazekii identified several putative virulence genes ${ }^{75}$. One example was pld, which, when expressed in a surrogate system, enabled Salmonella enterica subsp. enterica serovar Typhimurium to escape the phagosome $e^{76}$. Several years later, allelic exchange was used to replace wild-type pld of $R$. prowazekii with a mutated copy ${ }^{49}$. No differences in growth kinetics, rickettsial burden or ability to escape the phagosome were observed between the virulent wild-type $R$. prowazekii str. Madrid Evir and the pld mutant in vitro ${ }^{49}$. Nevertheless, in a guinea pig infection model, the $\Delta$ pld mutant was attenuated compared with the virulent $R$. prowazekii strain. Guinea pigs that were infected with the $\Delta$ pld mutant lost less weight and were afebrile compared with animals infected with virulent R. prowazekii ${ }^{49}$.

OmpA of $R$. rickettsii was considered a major virulence factor before the advent of genetic tools ${ }^{66,77}$. The original study relied on purified OmpA, which was shown to block the attachment of $R$. rickettsii when pre-incubated with host cells. Selective degradation and denaturation of OmpA also prevented R. rickettsii from adhering to host cells ${ }^{77}$. Uncertainties in regard to the function of OmpA remained, and group II intron technology was recently used to insert a $5^{\prime}$ premature stop codon into ompA of the highly virulent $R$. rickettsii str. Sheila Smith ${ }^{53}$. Unexpectedly, no differences in virulence between the wild-type and mutant strains were detected in either cell culture or the guinea pig infection mode ${ }^{53}$, refuting the notion that OmpA is required for, or enhances, the invasion and disease pathogenesis of R. rickettsii.

Advances in genetic tools have aided in clarifying the function of Chlamydia protease-like activity factor (CPAF). Following its identification, CPAF was shown to cleave host transcription factors that control the expression of major histocompatibility complex (MHC) class I and class $\mathrm{II}^{78}$, which ultimately leads to evasion of the immune system and undetected infection with Chlamydia spp. ${ }^{78}$. Subsequent studies reported that many other host proteins were cleaved by $\mathrm{CPAF}^{79}$. However, as genetic tools were unavailable, recombinant and ectopically expressed CPAF were used for validation of these substrates $^{79}$. Thirteen years after the initial discovery of CPAF, a C. trachomatis mutant with defects in CPAF expression was generated ${ }^{80}$. Using this mutant, several of the phenotypes ascribed to CPAF were shown to occur independently of CPAF. Specifically, CPAF did not affect Golgi fragmentation, NF- $\kappa \mathrm{B}$ activation, inhibition of apoptosis and protection from reinfection ${ }^{80}$. CPAF was described to cleave vimentin, which is an intermediate filament that surrounds the inclusion, and nuclear lamin-associated protein 1 (LAP1; also known as TOR1AIP1) following the disruption of the membrane ${ }^{80}$.
CPAF was also required for the optimal development of elementary bodies ${ }^{80}$ and has unanticipated roles in infection, including in the regulation of T3SS effectors and bacterial survival in the lower genital tract ${ }^{81,82}$. Despite these new findings, other questions in regard to the role of $\mathrm{CPAF}$ in infection remain; for example, whether CPAF is secreted into the host cytosol or is sequestered in its active form in the inclusion ${ }^{83}$. Overall, genetic tools have enabled the chlamydial and rickettsial fields to correct artefactual results, which benefits researchers and the public alike, as new therapeutics and vaccines against obligate pathogens are desperately needed.

\section{Concluding remarks}

Much has been achieved in the genetic engineering of obligate intracellular bacteria since the first transformation of C. trachomatis $^{3}$ (FIG. 1). The genetic toolbox for these organisms has grown to include transformation protocols; rickettsial, chlamydial and Coxiella shuttle vectors; random mutagenesis systems; and the ability to generate targeted knockouts through allelic exchange, group II intron technology and FRAEM (FICS 2,3). Despite these advances, limitations still impede the complete application of genetic tools. Chiefly among these is the lack of axenic media, which enables host-free growth of obligate intracellular bacteria. Challenges due to the intracellular lifestyle of obligate intracellular bacteria, tedious selection methods and the inability to efficiently obtain clonal populations limit the practicality of genetic manipulation. Routine complementation of mutagenized genes by expressing the wild-type gene under the control of its own promoter is still technically demanding. Nonetheless, the future of genetic manipulation of obligate intracellular bacteria is promising, as highlighted using the example of C. burnetii.

C. burnetii was strictly classified as an obligate intracellular bacterium until the formulation of an axenic medium that enabled its host-free cultivation in the laboratory ${ }^{4}$. This landmark development was facilitated through advances in genome sequencing and systematic refinements to the growth medium. No defect in infectivity has been shown for C. burnetii extracellular growth compared with host-cell cultivated bacteria ${ }^{4}$. C. burnetii grows much faster in axenic medium than in host cells and forms colonies, which enables selection and the subsequent expansion of clonal populations. Instead of several months, transformation experiments now take less than three weeks to complete, which has accelerated the development of genetic tools available to modify the genome of C. burnetii ${ }^{4,5}$ (FIG. 1). These genetic tools and the study of the metabolic requirements of C. burnetii have provided important insights into the composition and availability of nutrients in the parasitophorous vacuole.

One promising avenue of research is the rickettsial type IV secretion system (T4SS). Members of the Rickettsiales encode a vir-type P-type IV secretion system $(r v h)$ and use this molecular scaffold, in part, to remodel the host environment ${ }^{84-86}$. The functional characterization of $r v h$, including the identification of substrates, has been hampered by the inability to easily generate 
Rvh-deficient species in the Rickettsiales. Duplications and repeats in $r v h$ seem to have persisted despite evolutionary pressure towards genome reduction; thus, these features may be evidence of an alternative function or, conversely, a regulatory circuit ${ }^{86}$. We anticipate that mechanistic characterization of $r v h$, aided by mutagenesis using the TargeTron system or random approaches, will shed light onto rickettsial pathogenesis, virulence and host-pathogen interactions.

Another key question in the biology of obligate intracellular bacteria is how Chlamydia spp. and members of the Anaplasmataceae establish and protect their intracellular niches. Inc proteins that are secreted by Chlamydia spp. mediate these processes ${ }^{58}$. The functional relevance of each Inc protein, how they are regulated and in what manner they contribute to the establishment of this unique organelle, remain poorly understood. Although few Anaplasmataceae effectors have been characterized, some encode ankyrin repeat motifs that are common in eukaryotes and may have implications for virulence ${ }^{87}$. Ankyrin repeats mediate protein-protein interactions, provide scaffolding for signalling pathways and localize molecules to subcellular compartments in eukaryotes ${ }^{8}$. Ankyrin-repeat containing proteins (Anks) in A. phagocytophilum and E. chaffeensis were shown to mediate host epigenetic changes to promote bacterial survival ${ }^{69,89}$. Elucidating the function of conserved Anks in obligate intracellular bacteria can provide insight into host signalling pathways. Furthermore, characterizing mediators of inclusion development through genomic manipulation of obligate intracellular bacteria will reveal basic cell biology principles and 'druggable' targets.
Iron is an essential and limiting nutrient for both hosts and pathogens ${ }^{90}$. Remarkably, little is known about iron homeostasis in obligate intracellular bacteria, including sources, mechanisms of uptake and regulation during colonization of the mammalian host and the arthropod vector. A. marginale, a bovine pathogen that is transmitted by hard ticks, parasitizes erythrocytes. No iron regulatory elements, enzyme systems to degrade haem, surface receptors or siderophores have been identified in the genome of $A$. marginale ${ }^{91}$. Only a single orthologue to a bacterial transferrin (ferric binding protein $\mathrm{A}(\mathrm{FbpA})$ ), a permease $(\mathrm{FbpB})$, and a transporter that is thought to supply energy through ATP hydrolysis $(\mathrm{FbpC})$ have been found ${ }^{92}$. Unlike similar iron transport systems, these genes are not encoded in a single operon, but rather are dispersed throughout the genome of $A$. marginale ${ }^{93,94}$. How this minimal machinery is used for iron uptake and homeostasis is unknown, but routine targeted genetic manipulation could improve our understanding of bacterial iron acquisition.

The development of novel genetic tools, especially for members of the Rickettsiales, will be crucial to discover novel paradigms in microbial pathogenesis and immunity. As mutants of Chlamydia spp., Rickettsia spp., Orientia spp., Anaplasma spp., Ehrlichia spp. and Coxiella spp. are characterized, the research community will learn more about the fascinating lifestyles of these organisms. Although formerly thought of as just an exotic group of bacteria, the unfolding story of genetic engineering in obligate intracellular bacteria highlights the importance of intellectual curiosity, ingenuity and persistence to overcome scientific obstacles.
1. Vance, R. E. Immunology taught by bacteria. J. Clin. Immunol. 30, 507-511 (2010).

2. Shaw, D. K., McClure, E. E., Wang, X. ¿ Pedra, J. H. F. Deviant behavior: tick-borne pathogens and inflammasome signaling Vet. Sci. 3, 27 (2016).

3. Tam, J. E., Davis, C. H. \& Wyrick, P. B. Expression of recombinant DNA introduced into Chlamydia trachomatis by electroporation. Can. J. Microbiol. 40 , 583-591 (1994).

4. Omsland, A. \& Heinzen, R. A. Life on the outside the rescue of Coxiella burnetii from its host cell. Annu. Rev. Microbiol. 65, 111-128 (2011). This review describes the development of an axenic medium for $C$. burnetii, which enabled the rapid development of genetic tools for this microorganism.

5. Beare, P. A., Sandoz, K. M., Omsland, A., Rockey, D. D. \& Heinzen, R. A. Advances in genetic manipulation of obligate intracellular bacterial pathogens. Front. Microbiol. 2, 97 (2011).

6. O'Connor, T. J., Adepoju, Y., Boyd, D. \& Isberg, R. R. Minimization of the Legionella pneumophila genome reveals chromosomal regions involved in host range expansion. Proc. Natl Acad. Sci. USA 108,

14733-14740 (2011)

7. Beare, P. A. Genetic manipulation of Coxiella burnetii. Adv. Exp. Med. Biol. 984, 249-271 (2012).

8. Kokes, M. et al. Integrating chemical mutagenesis and whole-genome sequencing as a platform for forward and reverse genetic analysis of Chlamydia. Cell Host Microbe 17, 716-725 (2015).

This article reports the generation of a large-scale chemical mutagenesis library for $\boldsymbol{C}$. trachomatis serovar L2 and provides a systematic framework for screening mutants.

9. Sixt, B. S. et al. The Chlamydia trachomatis inclusion membrane protein $\mathrm{CpoS}$ counteracts STING-mediated cellular surveillance and suicide programs. Cell Host Microbe 21, 113-121 (2017).

This study describes a chlamydial effector that was discovered through chemical mutagenesis, verified through multiple genetic tools and shed light on the basic processes of eukaryotic host cell death

10. Lamason, R. L. et al. Rickettsia Sca4 reduces vinculinmediated intercellular tension to promote spread. Cell 167 670-683 e10 (2016).

This article reports the mechanism of intercellular spread for $R$. parkeri and the first published case of complementation of a rickettsial mutant with a shuttle vector.

11. Wang, Y. et al. Development of a transformation system for Chlamydia trachomatis: restoration of glycogen biosynthesis by acquisition of a plasmid shuttle vector. PLoS Pathog. 7, e 1002258 (2011). This protocol establishes a robust transformation method for C. trachomatis serovar L2 that is currently used extensively by the field.

12. Rachek, L. I., Tucker, A. M., Winkler, H. H. \& Wood, D. O. Transformation of Rickettsia prowazekii to rifampin resistance. J. Bacteriol. 180, 2118-2124 (1998).

13. Felsheim, R. F. et al. Transformation of Anaplasma phagocytophilum. BMC Biotechnol. 6, 42 (2006).

This study describes the generation of a Himar transposon mutagenesis library for $A$. phagocytophilum.

14. Long, S. W., Whitworth, T. J., Walker, D. H. \& Yu, X.-J. Overcoming barriers to the transformation of the genus Ehrlichia. Ann. NY Acad. Sci. 1063, 403-410 (2005).

15. Cheng, C. et al. Targeted and random mutagenesis of Ehrlichia chaffeensis for the identification of genes required for in vivo infection. PLoS Pathog. 9 e1003171 (2013).

16. Mishra, M. K., Gêrard, H. C., Whittum-Hudson, J. A., Hudson, A. P. \& Kannan, R. M. Dendrimer-enabled modulation of gene expression in Chlamydia trachomatis. Mol. Pharm. 9, 413-421 (2012).

17. Gérard, H. C. et al. Dendrimer-enabled DNA delivery and transformation of Chlamydia pneumoniae. Nanomedicine 9, 996-1008 (2013).
18. Oki, A T et al. Dendrimer-enabled transformation of Anaplasma phagocytophilum. Microbes Infect. 17, 817-822 (2015)

19. Thomas, N. S., Lusher, M., Storey, C. C. \& Clarke, I. N. Plasmid diversity in Chlamydia. Microbiology 143 1847-1854 (1997).

20. Seth-Smith, H. M. B. et al. Co-evolution of genomes and plasmids within Chlamydia trachomatis and the emergence in Sweden of a new variant strain. BMC Genomics 10, 239 (2009).

21. Agaisse, H. \& Derré, I. A. C. trachomatis cloning vector and the generation of $C$. trachomatis strains expressing fluorescent proteins under the control of a C. trachomatis promoter. PLoS ONE 8, e57090 (2013).

22. Bauler, L. D. \& Hackstadt, T. Expression and targeting of secreted proteins from Chlamydia trachomatis. J. Bacteriol. 196, 1325-1334 (2014).

23. Wickstrum, J., Sammons, L. R., Restivo, K. N. \& Hefty, P. S. Conditional gene expression in Chlamydia trachomatis using the Tet system. PLOS ONE 8, e76743 (2013)

24. Bastidas, R. J. \& Valdivia, R. H. Emancipating Chlamydia: advances in the genetic manipulation of a recalcitrant intracellular pathogen Microbiol. $\mathrm{Mol}$. Biol. Rev. 80, 411-427 (2016)

25. Sixt, B. S. \& Valdivia, R. H. Molecular genetic analysis of Chlamydia species. Annu. Rev. Microbiol. 70, 179-198 (2016)

26. Mueller, K. E. \& Fields, K. A. Application of $\beta$-lactamase reporter fusions as an indicator of effector protein secretion during infections with the obligate intracellular pathogen Chlamydia trachomatis. PLOS ONE 10, e0135295 (2015).

27. Mueller, K. E., Wolf, K. \& Fields, K. A. Gene deletion by fluorescence-reported allelic exchange mutagenesis in Chlamydia trachomatis. mBio 7, e01817-15 (2016).

28. Binet, R. \& Maurelli, A. T. Transformation and isolation of allelic exchange mutants of Chlamydia psittaci using recombinant DNA introduced by electroporation. Proc. Natl Acad. Sci. USA 106, 292-297 (2009). 
29. Burkhardt, N. Y. et al. Development of shuttle vectors for transformation of diverse Rickettsia species. PLOS ONE 6, e29511 (2011)

This article reports the generation of shuttle vectors that can be used to ectopically express proteins in diverse Rickettsia spp.

30. Wood, D. O. et al. Establishment of a replicating plasmid in Rickettsia prowazekii. PLOS ONE 7, e34715 (2012).

31. Hauptmann, M. et al. GFPuv-expressing recombinant Rickettsia typhi: a useful tool for the study of pathogenesis and $C D 8^{+} \mathrm{T}$ cell immunology in Rickettsia typhi infection. Infect. Immun. http://dx.doi.org/10.1128/IAl.00156-17 (2017).

32. Million-Weaver, S. \& Camps, M. Mechanisms of plasmid segregation: have multicopy plasmids been overlooked? Plasmid 75, 27-36 (2014).

33. Riley, S. P., Macaluso, K. R. \& Martinez, J. J. Electrotransformation and clonal isolation of Rickettsia species. Curr. Protoc. Microbiol. 39 3A.6.1-3A.6.20 (2015)

34. Riley, S. P. et al. Nonselective persistence of a Rickettsia conorii extrachromosomal plasmid during mammalian infection. Infect. Immun. 84, 790-797 (2016).

35. Oliver, J. D., Burkhardt, N. Y., Felsheim, R. F., Kurtti, T. J. \& Munderloh, U. G. Motility characteristics are altered for Rickettsia bellii transformed to overexpress a heterologous rickA gene. Appl. Environ. Microbiol. 80, 1170-1176 (2014).

36. Chen, C. et al. Large-scale identification and translocation of type IV secretion substrates by Coxiella burnetii. Proc. Natl Acad. Sci. USA 107, 21755-21760 (2010)

37. Voth, D. E. et al. The Coxiella burnetii cryptic plasmid is enriched in genes encoding type IV secretion system substrates. J. Bacteriol. 193, 1493-1503 (2011).

38. Moffatt, J. H., Newton, P. \& Newton, H. J. Coxiella burnetii: turning hostility into a home. Cell. Microbiol. 17, 621-631 (2015)

39. Lampe, D. J., Churchill, M. E. \& Robertson, H. M. A purified mariner transposase is sufficient to mediate transposition in vitro. EMBO J. 15, 5470-5479 (1996).

40. Beare, P. A et al. Characterization of a Coxiella burnetii ftsZ mutant generated by Himar 1 transposon mutagenesis. J. Bacteriol. 191, 1369-1381 (2009).

41. Reed, S. C., Lamason, R. L., Risca, V. I., Abernathy, E. $\&$ Welch, M. D. Rickettsia actin-based motility occurs in distinct phases mediated by different actin nucleators. Curr. Biol. 24, 98-103 (2014).

42. Liu, Z.-M., Tucker, A. M., Driskell, L. O. \& Wood, D. O. Mariner-based transposon mutagenesis of Rickettsia prowazekii. Appl. Environ. Microbiol. 73, 6644-6649 (2007).

43. Crosby, F. L. et al. Knockout of an outer membrane protein operon of Anaplasma marginale by transposon mutagenesis. BMC Genomics 15, 278 (2014).

44. Felsheim, R. F. et al. Transformation of Anaplasma marginale. Vet. Parasitol. 167, 167-174 (2010).

45. Rodolakis, A. In vitro and in vivo properties of chemically induced temperature-sensitive mutants of Chlamydia psittaci var. ovis: screening in a murine model. Infect. Immun 42, 525-530 (1983).

46. Kari, L. et al. Generation of targeted Chlamydia trachomatis null mutants. Proc. Natl Acad. Sci. USA 108, 7189-7193 (2011).

47. Brothwell, J. A. et al. Interrogating genes that mediate Chlamydia trachomatis survival in cell culture using conditional mutants and recombination. J. Bacteriol. 198, 2131-2139 (2016).

48. Nguyen, B. D. \& Valdivia, R. H. Virulence determinants in the obligate intracellular pathogen Chlamydia trachomatis revealed by forward genetic approaches. Proc. Natl Acad. Sci. USA 109, 1263-1268 (2012).

This study details the chemical mutagenesis procedure for Chlamydia trachomatis serovar L2 and describes the linkage analysis conducted to link mutation and phenotype.

49. Driskell, L. O. et al. Directed mutagenesis of the Rickettsia prowazekii pld gene encoding phospholipase D. Infect. Immun. 77, 3244-3248 (2009).

50. Suhan, M. L., Chen, S. Y. \& Thompson, H. A. Transformation of Coxiella burnetii to ampicillin resistance. J. Bacteriol. 178, 2701-2708 (1996).

51. Lambowitz, A. M. \& Zimmerly, S. Group II introns: mobile ribozymes that invade DNA. Cold Spring Harb. Perspect. Biol. 3, a003616 (2011).
52. Johnson, C. M. \& Fisher, D. J. Site-specific, insertional inactivation of incA in Chlamydia trachomatis using a group II intron. PLoS ONE 8, e83989 (2013).

53. Noriea, N. F., Clark, T. R. \& Hackstadt, T. Targeted knockout of the Rickettsia rickettsii OmpA surface antigen does not diminish virulence in a mammalian model system. mBio 6, e00323-15 (2015). This article uses targeted mutagenesis to show that a classical virulence factor in $R$. rickettsii is not important for virulence.

54. Driskell, L. O., Tucker, A. M., Woodard, A., Wood, R. R. $\&$ Wood, D. O. Fluorescence activated cell sorting of Rickettsia prowazekii-infected host cells based on bacterial burden and early detection of fluorescent rickettsial transformants. PLOS ONE 11, e0152365 (2016)

55. Troyer, J. M., Radulovic, S. \& Azad, A. F. Green fluorescent protein as a marker in Rickettsia typh transformation. Infect. Immun. 67, 3308-3311 (1999).

56. Alzhanov, D. T., Suchland, R. J., Bakke, A. C. Stamm, W. E. \& Rockey, D. D. Clonal isolation of Chlamydia-infected cells using flow cytometry. J. Microbiol. Methods 68, 201-208 (2007).

57. Podgorny, O. V. et al. Isolation of single Chlamydiainfected cells using laser microdissection. J. Microbiol. Methods 109, 123-128 (2015)

58. Elwell, C., Mirrashidi, K. \& Engel, J. Chlamydia cell biology and pathogenesis. Nat. Rev. Microbiol. 14, 385-400 (2016)

59. Lutter, E. I., Martens, C. \& Hackstadt, T. Evolution and conservation of predicted inclusion membrane proteins in chlamydiae. Comp. Funct. Genomics 2012 , 362104 (2012)

60. Donaldson, J. G. \& Jackson, C. L. ARF family G proteins and their regulators: roles in membrane transport, development and disease. Nat. Rev. Mol. Cell Biol. 12, 362-375 (2011).

61. Alix, E. et al. The capping domain in RalF regulates effector functions. PLoS Pathog. 8, e1003012 (2012).

62. Rennoll-Bankert, K. E. et al. Which way in? The RalF Arf-GEF orchestrates Rickettsia host cell invasion. PLoS Pathog. 11, e1005115 (2015)

63. Kagan, J. C. \& Roy, C. R. Legionella phagosomes intercept vesicular traffic from endoplasmic reticulum exit sites. Nat. Cell Biol. 4, 945-954 (2002).

64. Prantner, D., Perkins, D. J. \& Vogel, S. N. AMPactivated kinase (AMPK) promotes innate immunity and antiviral defense through modulation of stimulator of interferon genes (STING) signaling. J. Biol. Chem. 292, 292-304 (2017).

65. Hare, D. N. et al. Membrane perturbation-associated $\mathrm{Ca}_{2}{ }^{+}$signaling and incoming genome sensing are required for the host response to low-level enveloped virus particle entry. J. Virol. 90, 3018-3027 (2015).

66. Sahni, S. K., Narra, H. P., Sahni, A. \& Walker, D. H. Recent molecular insights into rickettsial pathogenesis and immunity. Future Microbiol. 8, 1265-1288 (2013).

67. Gillespie, J. J. et al Secretome of obligate intracellular Rickettsia. FEMS Microbiol. Rev. 39, 47-80 (2015).

68. Park, H., Lee, J. H., Gouin, E., Cossart, P. \& Izard, T. The rickettsia surface cell antigen 4 applies mimicry to bind to and activate vinculin. J. Biol. Chem. 286, 35096-35103 (2011)

69. Rikihisa, Y. Anaplasma phagocytophilum and Ehrlichia chaffeensis: subversive manipulators of host cells. Nat. Rev. Microbiol. 8, 328-339 (2010).

70. Chen, G. et al. Anaplasma phagocytophilum dihydrolipoamide dehydrogenase 1 affects hostderived immunopathology during microbial colonization. Infect. Immun. 80, 3194-3205 (2012).

71. Henderson, B. \& Martin, A. Bacterial virulence in the moonlight: multitasking bacterial moonlighting proteins are virulence determinants in infectious disease. Infect. Immun. 79, 3476-3491 (2011)

72. Dumler, J. S. The biological basis of severe outcomes in Anaplasma phagocytophilum infection FEMS Immunol. Med Microbiol. 64, 13-20 (2012).

73. Cheng, C., Nair, A. D., Jaworski, D. C. \& Ganta, R. R. Mutations in Ehrlichia chaffeensis causing polar effects in gene expression and differential host specificities. PLoS ONE 10, e0132657 (2015).

74. Nair, A. D. et al. Attenuated mutants of Ehrlichia chaffeensis induce protection against wild-type infection challenge in the reservoir host and in an incidental host. Infect. Immun. 83, 2827-2835 (2015).

5. Zhang, J. Z., Hao, J. F., Walker, D. H. \& Yu, X. J. A mutation inactivating the methyltransferase gene in avirulent Madrid E strain of Rickettsia prowazekii reverted to wild type in the virulent revertant strain Evir. Vaccine 24, 2317-2323 (2006).

76. Whitworth, T., Popov, V. L., Yu, X. J., Walker, D. H. \& Bouyer, D. H. Expression of the Rickettsia prowazekii pld or tlyC gene in Salmonella enterica serovar Typhimurium mediates phagosomal escape. Infect. Immun. 73, 6668-6673 (2005).

77. Li, H. \& Walker, D. H. rOmpA is a critical protein for the adhesion of Rickettsia rickettsii to host cells. Microb. Pathog. 24, 289-298 (1998).

78. Zhong, G., Fan, P., Ji, H., Dong, F. \& Huang, Y. Identification of a chlamydial protease-like activity factor responsible for the degradation of host transcription factors. J. Exp. Med. 193, 935-942 (2001).

79. Chen, A. L., Johnson, K. A., Lee, J. K., Sutterlin, C. \& Tan, M. CPAF: a chlamydial protease in search of an authentic substrate. PLoS Pathog. 8, e 1002842 (2012).

This study shows that several substrates of a chlamydial protease are artefacts of sample processing.

80. Snavely, E. A. et al. Reassessing the role of the secreted protease CPAF in Chlamydia trachomatis infection through genetic approaches. Pathog. Dis. 71 336-351 (2014)

This article shows that several substrates of a chlamydial protease are artefactual through the use of a CPAF-processing-defective $C$. trachomatis serovar L2 strain.

81. Patton, M. J. et al. Chlamydial protease-like activity factor and type III secreted effectors cooperate in inhibition of p65 nuclear translocation. $\mathrm{mBio} 7$ e01427-16 (2016).

82. Yang, Z. et al. The Chlamydia-secreted protease CPAF promotes chlamydial survival in the mouse lower genital tract. Infect. Immun. 84, 2697-2702 (2016).

83. Bavoil, P. M. \& Byrne, G. I. Analysis of CPAF mutants: new functions, new questions (the ins and outs of a chlamydial protease). Pathog. Dis. 71, 287-291 (2014).

84. Dunning Hotopp, J. C. et al. Comparative genomics of emerging human ehrlichiosis agents. PLoS Genet. 2 e21 (2006).

85. Cho, N.-H. et al. The Orientia tsutsugamushi genome reveals massive proliferation of conjugative type IV secretion system and host-cell interaction genes. Proc. Natl Acad. Sci. USA 104, 7981-7986 (2007).

86. Gillespie, J. J. et al. The Rickettsia type IV secretion system: unrealized complexity mired by gene family expansion. Pathog. Dis. 74, ftw058 (2016).

87. Rikihisa, Y. \& Lin, M. Anaplasma phagocytophilum and Ehrlichia chaffeensis type IV secretion and Ank proteins. Curr. Opin. Microbiol. 13, 59-66 (2010).

88. Jernigan, K. K. \& Bordenstein, S. R. Ankyrin domains across the tree of life. PeerJ 2, e264 (2014).

89. Lina, T. T. et al. Hacker within! Ehrlichia chaffeensis effector driven phagocyte reprogramming strategy. Front. Cell. Infect. Microbiol. 6, 58 (2016).

90. Andrews, S. C., Robinson, A. K. \& RodriguezQuinones, F. Bacterial iron homeostasis. FEMS Microbiol. Rev. 27, 215-237 (2003).

91. Brayton, K. A. et al. Complete genome sequencing of Anaplasma marginale reveals that the surface is skewed to two superfamilies of outer membrane proteins. Proc. Natl Acad. Sci. USA 102, 844-849 (2005).

92. Dhungana, S. et al. The influence of the synergistic anion on iron chelation by ferric binding protein, a bacterial transferrin. Proc. Natl Acad. Sci. USA 100 3659-3664 (2003).

93. Khun, H. H., Deved, V., Wong, H. \& Lee, B. C. fbpABC gene cluster in Neisseria meningitidis is transcribed as an operon. Infect. Immun. 68, 7166-7171 (2000).

94. Doyle, C. K., Zhang, X., Popov, V. L. \& McBride, J. W. An immunoreactive 38-kilodalton protein of Ehrlichia canis shares structural homology and iron-binding capacity with the ferric ion-binding protein family. Infect Immun 73, 62-69 (2005).

95. Ge, Y. \& Rikihisa, Y. Subversion of host cell signaling by Orientia tsutsugamushi. Microbes Infect. 13, 638-648 (2011).

96. Beare, P. A., Larson, C. L., Gilk, S. D. \& Heinzen, R. A Two systems for targeted gene deletion in Coxiella burnetii. Appl. Environ. Microbiol. 78, 4580-4589 (2012)

97. van Schaik, E. J., Chen, C., Mertens, K., Weber, M. M. $\&$ Samuel, J. E. Molecular pathogenesis of the obligate intracellular bacterium Coxiella burnetii. Nat. Rev. Microbiol. 11, 561-573 (2013). 
98. Valbuena, G. \& Walker, D. H. Approaches to vaccines against Orientia tsutsugamushi. Front. Cell. Infect. Microbiol. 2, 170 (2013).

99. Menon, S. et al. Human and pathogen factors associated with Chlamydia trachomatis-related infertility in women. Clin. Microbiol. Rev. 28. 969-985 (2015).

100. Hu, V. H., Holland, M. J. \& Burton, M. J. Trachoma: protective and pathogenic ocular immune responses to Chlamydia trachomatis. PLoS Negl. Trop. Dis. 7, e2020 (2013).

101. Dumler, J. S., Madigan, J. E., Pusterla, N. \& Bakken, J. S. Ehrlichioses in humans: epidemiology, clinical presentation, diagnosis, and treatment. Clin. Infect. Dis. 45, S45-S51 (2007).

102. Stuen, S., Granquist, E. G. \& Silaghi, C. Anaplasma phagocytophilum - a widespread multi-host pathogen with highly adaptive strategies. Front. Cell. Infect. Microbiol. 3, 31 (2013).

103. Merhej, V., Angelakis, E., Socolovschi, C. \& Raoult, D. Genotyping, evolution and epidemiological findings of Rickettsia species. Infect. Genet. Evol. 25, 122-137 (2014).

104. Weitzel, T. et al. Endemic scrub typhus in South America. N. Engl. J. Med. 375, 954-961 (2016).

105. Newman, L. et al. Global estimates of the prevalence and incidence of four curable sexually transmitted Infections in 2012 based on systematic review and global reporting. PLOS ONE 10, e0143304 (2015).
106. Taylor, H. R., Burton, M. J., Haddad, D., West, S. \& Wright, H. Trachoma. Lancet 384, 2142-2152 (2014).

107. Sanchez, E., Vannier, E., Wormser, G. P. \& Hu, L. T. Diagnosis, treatment, and prevention of Lyme disease, human granulocytic anaplasmosis, and babesiosis: a review. JAMA 315, 1767-1777 (2016).

108. Dahlgren, F. S., Mandel, E. J., Krebs, J. W. Massung, R. F. \& McQuiston, J. H. Increasing incidence of Ehrlichia chaffeensis and Anaplasma phagocytophilum in the United States, 2000-2007. Am. J. Trop. Med. Hyg. 85, 124-131 (2011).

109. Kelly, D. J., Richards, A. L., Temenak, J., Strickman, D. $\S$ Dasch, G. A. The past and present threat of rickettsial diseases to military medicine and international public health. Clin. Infect. Dis. 34, S145-S169 (2002).

110. Richards, A. L. Worldwide detection and identification of new and old rickettsiae and rickettsial diseases. FEMS Immunol. Med. Microbiol. 64, 107-110 (2012).

111. Nakayama, K. et al. The whole-genome sequencing of the obligate intracellular bacterium Orientia tsutsugamushi revealed massive gene amplification during reductive genome evolution. DNA Res. 15, 185-199 (2008).

\section{Acknowledgements}

The authors apologize to those colleagues whose work could not be cited owing to the broad scope of the Review and space limitations. Work in the authors' laboratories was supported by the US National Institutes of Health (Institutional Training Grant T32AI007540 to E.E.M.; R01 Al072683, R56 Al1 23346 and R21 Al1 22014 to J.A.C.; R01 Al100759 to R.H.V.; U 19 AI084044 to P.M.B.; R01 AI070908 to R.R.G.; R01 Al020384 and R21 Al103272 to D.O.W.; R01 Al042792 to U.G.M. and K.A.B.; R01 AI072606 and R21 Al111086 to J.J.M.; R01 Al106859 and R21 Al115449 to J.W.M.; and R01 AI093653 and R01Al116523 to J.H.F.P.), the US Department of Agriculture (USDA-ARS 2090-32000-038-00D to S.M.N.), the Center of Excellence for Vector-Borne Diseases at Kansas State University (to R.R.G.) and the University of Maryland School of Medicine (to J.H.F.P.). The content of this Review is solely the responsibility of the authors and does not necessarily represent the official views of the US National Institute of Allergy and Infectious Diseases or the US National Institutes of Health.

\section{Competing interests statement}

The authors declare no competing interests.

\section{Publisher's note}

Springer Nature remains neutral with regard to jurisdictional claims in published maps and institutional affiliations.

SUPPLEMENTARY INFORMATION

See online article: $\underline{\mathrm{S} 1}$ (figure)

ALL LINKS ARE ACTIVE IN THE ONLINE PDF 